Title page

'Causation in a Timeless world?'

Name: Dr. Jonathan Tallant

Institution: Department of Philosophy, University of Nottingham, Nottingham, NG7 2RD, UK

Email: jonathan.tallant@nottingham.ac.uk 


\begin{abstract}
This paper is an attempt to answer the question, 'could there be causation in a timeless world?' My conclusion: tentatively, yes. The paper and argument have three parts. Part one introduces salient issues and spells out the importance of this (initially somewhat baroque seeming) line of investigation. Section two of the paper reviews recent arguments due to Baron and Miller (2015a), who argue in favor of the possibility of causation in a timeless world, and looks to reject their arguments developed there. Section three is a response to a response. In their (2015a), Baron and Miller also argue that an argument in favor of the possibility of causation at timeless worlds, that I put forward (Tallant, 2008), is an argument that fails. In section three, my response to Baron and Miller is that their argument against me succeeds, but that there is a nearby argument that we can appeal to in order to demonstrate the possibility of causation at timeless worlds.
\end{abstract}




\section{Timelessness and Causation}

Over the last 20-30 years, a number of arguments have been brought forward to motivate and defend positions that deny the reality of time (Barbour, 1994a, 1994b, 1999; Barbour and Isham 1999; Baron and Miller 2014; Deutsch 1997; Roveli 2004, 2007, 2009; Tallant 2008). These arguments have been developed from a range of concerns in physics and metaphysics. Following Baron and Miller (2015b: 2428), call such positions 'temporal error theories'. A fair number of these positions maintain not merely that it is possible for time to be unreal, but that, as a matter of actual fact, time is unreal; temporal error theory is true of the actual world.

Such error theories seem extremely radical and, at least so far, have proven unpopular. Baron and Miller (2015b), in the course of looking to determine what temporal error theory is, look to diagnose the cause of this unpopularity and, as such, some of the difficulties that must be faced by a proponent of a timeless theory of reality. Baron and Miller identify two broad reasons for the unpopularity of timeless accounts of reality. First, they claim (2015b: 2430) that we conceive of ourselves as agents and, in order for us to be agents, this requires that we: (i) persist through time, (ii) are causally efficacious; (iii) are capable of instituting change in the world. Second (2015b: 2430-1), they claim, our temporal phenomenology convinces us of the reality of time (and, by 'temporal phenomenology', they mean to include a range of experiences; of succession, of memory, and, perhaps, even of passage). Plainly, both (ii) and (iii), above, would prima facie seem to require the reality of causation. But, at least so goes the worry, prima facie causation is something that occurs in time. It requires the reality of time.

There seems to be a general shape to these concerns. There are a range of phenomena (the sense of ourselves as agents; our temporal phenomenology, etc.) that seem to obtain; that seem to play central roles in our picture of the world. If we are to endorse a temporal error theory, then we must find some way of reconstructing that picture, a picture within which causation seems to play a central role. But we must carry out that reconstruction in the absence of the reality of time (I borrow the language of 'reconstruction' in this context from Healy (2002)).

And it is here that the relevance and crucial importance of the current project becomes clear. If we cannot have causation in the absence of time, then this puts a significant obstacle in the 
path of reconstruction of this picture of the world. If, in contrast, we can preserve the reality of causation in the absence of time, then we will have made a significant step along the road to a defence of a temporal error theory. We will have made substantial inroads into this task of reconstructing our pre-theoretic picture of reality within a timeless world. That being so, the prospects for a timeless model of reality appear to be intimately connected to the possibility of causation at a such a timeless world. Given the interest in such models, the project of reconstructing causation at a timeless world is therefore of significant import.

Be that as it may, the initial prospects for such a reconstruction do not look all that promising. Again, it is very tempting to see causation as essentially temporal. Witness: intuitively, if I throw a ball at a window and cause it to break, then one event, e, my throwing, causes another event, e', the breaking. Of course, e precedes e'. And whilst there are those who think that causation can occur simultaneously (eg. Mumford and Anjum, 2011), for instance where a ball resting on a cushion causes an indentation in the surface of the cushion, note that this is still clearly a case of causation occurring in a scenario where time is real--for, or so I assume, if e occurs simultaneously with e', then we cannot credibly claim that time is unreal in such a scenario. Simultaneity, just like precedence, is a temporal relation. The proponent of causation at timeless worlds thus faces an uphill struggle. Against that backdrop, let us turn our attention to the details of Baron and Miller's arguments.

\section{Baron and Miller}

Let us now turn to the arguments developed by Baron and Miller (2015a) that purport to show that it is possible for there to be causation at timeless worlds. To do justice to their argument we need to get clear on some of their assumptions and how they see the shape of the dialectic. First, then, let's consider the assumptions.

First assumption: the (so-called) B-relations are both necessary and sufficient for the reality of time. That is, time is real at world iff a world is structured by the relations earlier than, later than, and simultaneous with (cf. 2015a: 28).

Second assumption: counterfactual dependence is sufficient for causation. That is, if event e occurs and event c occurs, and it is true that, 'if event e had not occurred, event c would not have occurred', then it's true that 'event e caused event c' (cf. 2015a: 28). Given certain 
specific classes of problematic counterfactual, this requires augmentation to restrict which kinds of counterfactual dependence get to count as causal dependence. Leaning on Lewis (1973, 1986a), Baron and Miller (2015a: 28) endorse the following view.

"[C]ausal counterfactuals are counterfactuals that hold between distinct events where events have the following features: they are (i) matters of contingent fact regarding properties localized to regions, (ii) predominantly intrinsic, (iii) capable of standing in relations of implication (e.g., the proposition <event x occurs> might entail <event $y$ occurs >), (iv) non-disjunctive, and (v) stand in parthood relations to other events: both directly and indirectly, by being parts of the regions that other events occupy. Two events, $\mathrm{x}$ and $\mathrm{y}$, are distinct when (a) $\mathrm{x}$ and $\mathrm{y}$ do not stand in relations of implication, and (b) $\mathrm{x}$ is not part of $\mathrm{y}$ or vice versa."

Call this thesis 'Distinctness'.

From here, Baron and Miller's argument is, structurally, very straightforward. First they look to show that there are (or at least could be) genuinely timeless physical laws that fully govern a possible world, without the addition of any temporal structure. Second, they show that, in such a scenario, counterfactual dependence would hold between distinct events. Third, they have in the background their claim that there is a species of counterfactual dependence between distinct events, causal counterfactual dependence, that suffices for causation. Thus, they conclude, causation can occur at a timeless world.

My view of what we should conclude is somewhat different. To give the reader a sense of things: First I endorse Baron and Miller's claim that there are (or at least could be) genuinely timeless physical laws that fully govern a possible world, without the addition of any temporal structure. Second, I argue that, in such a scenario, counterfactual dependence would hold between distinct events, but that this is a species of counterfactual dependence that we have good reason to think is not causal. In other words, careful consideration of a range of cases suggest that we ought to reject their second assumption: that counterfactual dependence is sufficient for causation. In 2.2 I will argue that the structure of Baron and Miller's case is non-causal. In 2.3 I suggest that we might in any case be able to accommodate that result within the counterfactual theory of causation. 
Thus, Baron and Miller's case gives us no reason to think that it is possible to have causation at a timeless world. Though reasonably simple in the abstract, the details of the argument involved are more complex. Let us now move to explore these details.

\subsection{Timeless physical theory}

Very roughly, here is the case (that I will give more formally in just a moment). One of the most fundamental equations in quantum mechanics is the Schrodinger equation. There is a time-independent version of this equation that includes no time variable. If this equation correctly describes the fundamental structure of a world, w, then (or so we will assume, following Baron and Miller) time is not real at w. w will lack any temporal structure. Nonetheless, we will still find at such a world particular distributions of what look like events. Thus, at some point in w, governed by the time-independent version of the Schrodinger equation, entangled systems will arise. At some points, such entangled systems will collapse due to a measurement of the system, and at some other points we will find a situation in which one particle of that entangled system, P1, is in a spin-up state, and the other particle of that entangled system, $\mathrm{P} 2$, is in a spin-down state.

Slightly more formally, here is the case. Begin with a simple quantum system, Q, describable by the time-independent Schrödinger wave-equation:

Following Baron and Miller (2015a: 32), assume that Q has the following features:

(i) Q is a two-particle system, consisting of the particles P1 and P2

(ii) P1 and P2 are entangled

(iii) P1 and P2 possess a single quantum property: spin

(iv) There are two possible combinations of spin properties for P1 and P2:
a. $\quad\{|\uparrow>,| \downarrow>\}$ (i.e., P1 has spin up and P2 has spin down)
b. $\quad$ (b) $\{|\downarrow>,| \uparrow>\}$ (i.e., P1 has spin down and P2 has spin up)

As they then go on to point out: 
Now, imagine a simplified world, wD, in which (i) there is a space-evolving law governed by the time-independent Schrödinger wave-equation; (ii) there are just two slices S 1 and S 2 in w (iii) there are no B-relations between S 1 and S 2 or, indeed, in the world in general and (iii) P 1 and P 2 exist at S 1 and are such that both particles are entangled and have determinate quantum properties. In such a world, information about $\mathrm{P} 1$ and the quantum system in which it is embedded provides full information about (or assign probabilities to descriptions of) $\mathrm{P} 2$ at $\mathrm{S} 1$. Moreover, in $\mathrm{w} \mathrm{D}$, a range of counterfactual conditionals are true. If in $\mathrm{wD} \mid \mathrm{P} 1 \uparrow>$, then: $\sim \mathrm{P} 1 \uparrow>\square \rightarrow \sim \mid \mathrm{P} 2$ $\downarrow>$ will be true. That's because the closest possible worlds to wD in which $\sim \mid \mathrm{P} 1 \uparrow>$ holds, will be a world with the same laws and, in particular, the same space-evolving law as specified in (i). Hence, in that world, P 1's spin determines, across space, the spin of P 2 and so if P 1 is not spin up, then P 2's spin will not be down. Similarly, if $\mid \mathrm{P} 1 \downarrow>$ then it follows that: $\sim \mathrm{P} 1 \downarrow>\square \rightarrow \sim \mid \mathrm{P} 2 \uparrow>$. Note that the laws in this case are symmetrical. We can just as easily determine information about P 1 from $\mathrm{P} 2$. As such, if $\mid \mathrm{P} 2 \downarrow>$, then $\sim|\mathrm{P} 2 \downarrow>\square \rightarrow \sim| \mathrm{P} 1 \uparrow>$ and if $\mid \mathrm{P} 2 \uparrow>$, then $\sim|\mathrm{P} 2 \uparrow>\square \rightarrow \sim| \mathrm{P}$ $1 \downarrow>$. (2015a: 33).

In short: were the event of $\mathrm{P} 1$ having spin up to obtain, then the event of $\mathrm{P} 2$ having spin down would obtain. Similar remarks hold were the event of P1's having spin down to obtain (and because the system is symmetrical, similar remarks hold if we place the spin of P2 in the antecedent of the counterfactual conditional). And, say Baron and Miller, since what we have here is counterfactual dependence (and since the event of P1 having spin up is distinct from the event of P2 having spin down, given Distinctness), so what we have here is causation.

I think that Baron and Miller are wrong about this case. My argument (2.2) shows that the structure of Baron and Miller's case is paradigmatically non-causal. In (2.3) I suggest that we can retain that result within the counterfactual theory of causation.

\subsection{Response to Baron and Miller}

Structurally, the case that Baron and Miller present us with is interesting. It can (loosely) be described as a case in which two entities (P1 and P2) are brought together and bound to one another, such that they are entwined (entangled). This entwining is then what generates 
counterfactual dependence. This is what occurs in the case where time is real. In the timeless case, we simply remove the first step: it's not that the two entities are brought together and then bound; that would require action over time. Simply, they are bound. The combination of their being in an entangled state and the laws of nature ensure that they are bound to one another. Again, though, because they are entwined by this binding, we have counterfactual dependence.

As I say, that's all very loose and imprecise and not intended as anything so robust as an analysis. Rather, this loose description is intended to get us to a position where it's easy to see how to develop cases that are similar. And that is what I will do. In the next section I'll introduce a case that is similar to the entangled state case that Baron and Miller discuss. We have binding and hence entwining that leads to counterfactual dependence. I'll suggest, though, that despite the similarities, there is no causation in the case I describe. For reasons that I'll discuss, I think that this should lead us to conclude that there is no causation in the entangled state case that Baron and Miller discuss. I'll also use the first case and ensuing discussion to generate a further case that also puts pressure on their claim that what the entangled case shows us is that we can have causation in a timeless world. Let us now consider the first case.

Consider two solid metal cylinders, both of $5 \mathrm{~cm}$ diameter and $50 \mathrm{~cm}$ length. One is red, the other is blue: call them (unimaginatively) RED and BLUE. These two cylinders are then brought together such that their ends are touching, and then soldered together so that they are fused very tightly and securely. The red and blue cylinders are now bound to one another and collectively constitute a multi-coloured rod. We can then apply a rotational force to the (whole length of the) rod such that it will rotate with angular velocity, v. Let us suppose that the force is applied at $\mathrm{t}$. A little later-perhaps just a few seconds later-, at $\mathrm{t}^{*}$, the rod has angular velocity v'. At $\mathrm{t}^{*}$, the following counterfactual is true: were RED to have an angular velocity other than v', then BLUE would have an angular velocity other than v' (mutatis mutandis for what would be the case were BLUE to have a velocity other than v').

First things first: though there is counterfactual dependence of BLUE's angular velocity on RED's (and vice versa), this is not causation. At least, that is quite clearly the intuitive verdict. Where we have a rod (or any other object for that matter) that is rotating, we do not (ever) assert that the one half rotating is the cause of the other half rotating. Second things 
second: the case is structurally similar to the case discussed by Baron and Miller. We have two entities (RED and BLUE) that are (in a sense) entwined. This entwining generates counterfactual dependence between the properties (the angular velocity) of the two entities. Nonetheless, it is perfectly clear in the RED-BLUE case that we do not have causation. If we do not have causation in the RED-BLUE case, then (by parity of reasoning) we ought not to say that we have causation in Baron and Miller's case, for the two cases are structurally similar.

At this stage, I want to pause and consider an objection. Doing so allows me to get to the heart of the matter. Here is the thought. There is a reason that we do not have causation in the RED-BLUE case. This reason does not extend to the case of quantum entanglement in a timeless world. That being so, we lose our reason for thinking that two cases are relevantly similar. Sure, we have reason to think that RED-BLUE is not causal, but that tells us nothing of interest about entanglement in a timeless world.

Here is the argument: the counterfactual 'were RED to have an angular velocity other than v', then BLUE would have an angular velocity other than v' ' is true. So much must be admitted. But is true only because it invokes a back-tracking counterfactual. The following gives the pattern of reasoning.

(1) 'were RED to have an angular velocity other than v', then BLUE would have an angular velocity other than $\mathrm{v}$ " is true

(2) (1) is true because: were RED and BLUE to have different angular velocities, then they would not have been soldered together in the first place

The counterfactual conditional contained within (2) is a backtracking conditional and, albeit somewhat by fiat, when introducing the counterfactual theory of causation, Lewis (1986a) explicitly states that no such counterfactual is to be regarded as causal. So, sure: (1) is true. But (1) is true only because (2) holds, and (2) is explicitly a non-causal counterfactual. In that sense, then, it's tempting to say that there is no causation here because what underwrites the judgement that (1) is not causal is that it holds only because an explicitly non-causal counterfactual holds. 
How does that help Baron and Miller? Well, in the case of quantum entanglement that occurs in the actual world, the situation may appear exactly parallel.

(1)* Were particle P1 to be spin up, then particle P2 would be spin down

$(2)^{*}(1)^{*}$ is true because: were P1 and P2 to have the same spin values, then they would not have been entangled

But — and this is important - in the timeless case, this cannot be quite right. In the timeless case that Baron and Miller describe, their example is set up in such a way as to not involve backtracking counterfactuals in this kind of way.

By way of reminder:

Now, imagine a simplified world, wD, in which (i) there is a space-evolving law governed by the time-independent Schrödinger wave-equation; (ii) there are just two Slices S 1 and S 2 in w (iii) there are no B-relations between S 1 and S 2 or, indeed, in the world in general and (iii) P 1 and P 2 exist at S 1 and are such that both particles are entangled and have determinate quantum properties. In such a world, information about $\mathrm{P} 1$ and the quantum system in which it is embedded provides full information about (or assign probabilities to descriptions of) $\mathrm{P} 2$ at $\mathrm{S} 1$. Moreover, in $\mathrm{w} \mathrm{D}$, a range of counterfactual conditionals are true. If in $\mathrm{wD} \mid \mathrm{P} 1 \uparrow>$, then: $\sim|\mathrm{P} 1 \uparrow>\square \rightarrow \sim| \mathrm{P} 2$ $\downarrow>$ will be true. (2015a: 33)

Crucially, determining the spin of one particle by determining the spin of the other can be achieved via the wave equation. ${ }^{1}$ That being so, there is no backtracking counterfactual here, we just have two particles in an entangled state; the spin of one particle and a law of nature. But there can't be backtracking counterfactuals about the timeless world, for there is (to put it a little crudely) no back into which we may track. Less idiomatically: since there is no time, there is no past for the counterfactual to backtrack into.

So (goes the thought): even if I'm right and in the RED-BLUE case we don't have causation, that we don't have causation is due to the fact that case involves backtracking

\footnotetext{
${ }^{1}$ Thanks to a referee for this thought. It (helpfully) unpicked most of what I originally wanted to say.
} 
counterfactuals. This case, the case that that Baron and Miller present us with that involves timeless worlds, doesn't (indeed, can't) invoke backtracking counterfactuals. That being so, we can't make the move to say that the Baron and Miller case doesn't involve causation because it involves a backtracking counterfactual; simply, it involves no such counterfactual. And if there is no backtracking counterfactual explanation of why the counterfactual is true, then that marks a very significant difference between RED-BLUE and the case of quantum entanglement at a timeless world. Such differences should make us wary. We ought not to infer conclusions about the entanglement case based on cases like RED-BLUE.

Perhaps. I think that such a line might actually be resisted by arguing that RED-BLUE fails describe a causal scenario for reasons other than those that invoke backtracking counterfactuals. But set that to one side. I think it's straightforward enough to develop a revised version of the RED-BLUE scenario that is threatening to Baron and Miller's claims.

As before, suppose that RED and BLUE are brought together such that their ends are touching, and then soldered together so that they are fused very tightly. They are now bound to one another and collectively constitute a multi-coloured rod. We can then apply a rotational force to the rod and it will rotate with angular velocity, v. As before, let us now consider the rotational velocity of the rod at $t^{*}$. Now things get more complex. Note, first, that following good Lewisian strictures, we might endorse a principle of modal recombination. Roughly:

[A]nything can coexist with anything else, at least provided they occupy distinct spatiotemporal positions. Likewise anything can fail to coexist with anything else (1986b: 88).

Importantly, though trivially, because $t$ is not identical to $t^{*}, t$ and $t^{*}$ occupy distinct spatiotemporal positions. Thus, the rod (with angular velocity v') can exist at $\mathrm{t}^{*}$, at a possible world that does not include $t$. This is what the principle of recombination allows us. And since velocity is often taken to hold at an instant there should be no barrier to this case being regarded as possible. ${ }^{2}$ Thus, call the possible world that consists of just $t^{*}, \mathrm{wt}^{*}$. It's true, at $\mathrm{wt}^{*}$, that the rod rotates with angular velocity v'. It's also true that RED rotates with angular

\footnotetext{
${ }^{2}$ See Carroll (2002) inter alia for discussion.
} 
velocity v' and that BLUE rotates with angular velocity v'. Crucially, it's also true that at wt* that: were RED to have an angular velocity other than v', then BLUE would have an angular velocity other than v'.

But, as before, it seems obviously wrong to say that RED's having angular velocity v' is a cause of BLUE's having angular velocity v'. And, of course, there is no scope for appealing to any backtracking counterfactuals to explain this phenomena at wt*, because wt* has no history. Call this revised case RED-BLUE* Once again, then, we have an objection to Baron and Miler. Their case is just like RED-BLUE*. RED-BLUE* is not causal. Their case is not causal.

I then need to be clear on three points. First, RED-BLUE* is not a case of causation. So much seems wholly obvious. Second, RED-BLUE* is of the same form as the entangled particles case described by Baron and Miller RED-BLUE* presents a useful comparison with the case Baron and Miller generate. RED-BLUE* isn't causal. It is of the same form as Baron and Miller's case. That being so, Baron and Miller's case isn't causal either.

Third, and slightly more tentatively, I think that there is an intuitive revision that we can (and perhaps should) make to the counterfactual analysis of causation. This revision accommodates the insight that RED-BLUE* isn't causal, but predicts that Baron and Miller's case isn't causal, either.

\subsection{Non-causal counterfactuals revisited}

We need to revisit the Lewisian criteria as to which counterfactuals are to be regarded as causal counterfactuals. Here are Baron and Miller (2015a: 34)

Lewis (1986[a]) argues that causal counterfactuals are counterfactuals that hold between distinct events where events have the following features: they are (i) matters of contingent fact regarding properties localized to regions, (ii) predominantly intrinsic, (iii) capable of standing in relations of implication (e.g., the proposition <event x occurs> might entail <event y occurs>), (iv) non-disjunctive, and (v) stand in parthood relations to other events: both directly and indirectly, by being parts of the regions that other events occupy. Two events, $x$ and $y$, are distinct when (a) $x$ and $y$ do not stand in relations of implication, and (b) $\mathrm{x}$ is not part of $\mathrm{y}$ or vice versa. 
I agree with Baron and Miller that the case of spin in quantum mechanics is a paradigm case of an intrinsic property. However, I think that it's clear enough that the rod case should motivate a revision to the Lewisian restriction on which counterfactuals are to be regarded as causal. The next question: what kind of restriction?

Schematically, the RED-BLUE* case has the following structure. A fusion of $x$ (RED) and $y$ (BLUE), call it $\mathrm{z}$ (the rod), has a particular property, $\mathrm{P}$ (the rotational velocity). We find ourselves in a position where, z's instantiating P and x's instantiating P determines, or fixes, the fact that $y$ has a particular property - in the rod case, $P .{ }^{3}$ More perspicuously: it's necessary that: if $\mathrm{x}$ is a part of $\mathrm{z}$ and $\mathrm{y}$ is a part of $\mathrm{z}$, and $\mathrm{x}$ and $\mathrm{z}$ both instantiate $\mathrm{P}$, that $\mathrm{y}$ 's rational velocity property is fixed. That's just the way this $P$ behaves. Note, then, that: if $y$ instantiated a (rotational velocity property other than $\mathrm{P}$ ), then both $\mathrm{x}$ and $\mathrm{z}$ would instantiate a (rotational velocity property other than P). Thus we have counterfactual dependence of y's rotational velocity on the properties of $\mathrm{x}$ and $\mathrm{z}$. But, again, this is not causal.

Consider another case: if a table, $\mathrm{z}$, is red (all over) and $\mathrm{x}$ (a part of the table) is red, then $\mathrm{y}$ (another part of the table) cannot be anything other than red. And: If $\mathrm{z}$ is red all over, then: were it the case that $\mathrm{x}$ is not red, then it would not be the case that $\mathrm{y}$ is red. Of course, we do not take that to show that x's being red is the cause of y's being red. Thus we have noncausal counterfactual dependence of y's colour property on the properties of $\mathrm{x}$ and $\mathrm{z}$.

It seems reasonably clear roughly what is going on in these cases. It is the properties of the system, in concert with a property of some part of the system, that are in some way determining the property of another part of the system. In those cases, counterfactual dependence does not lead to causation. Slightly more formally, the idea is this. If there is some overarching property, F, of a system, S, that independently or in concert with some part of the system, s1, and its property, $\mathrm{G}$, serves to necessitate that the property, $\mathrm{H}$, is instantiated, by some part of the system, s2, that is not identical to $\mathrm{s} 1$, then the instantiation of $\mathrm{F}$ and $\mathrm{G}$ (individually or jointly) are not among the causes of s2 instantiating $\mathrm{H}$.

\footnotetext{
${ }^{3}$ Admittedly in the rod case it seems natural to think that $\mathrm{x}$ is idle; what does the explanatory work is that $\mathrm{z}$ has the property. Nonetheless, if z's instantiating P necessitates y's instantiating P, then it follows (albeit trivially) that $x$ and $z$ 's instantiating $P$ necessitates $y$ 's instantiating $P$.
} 
A case may serve to illustrate why this is intuitive. A coin (a system) has a property of having two sides that face in opposite directions. If a coin (idealised, such that it cannot rest on its edge) lands heads side up, that serves to fix/determine that the other side of the coin is face down. ${ }^{4}$ In other words, it fixes that the coin lands tails side down. This allows us to generate the following true counterfactual: were the coin to not land heads up, then it would not have landed tails down. That's a true counterfactual. But that does not mean that the coin landing heads side up is a cause of the coin landing tails side down. Further, even if we consider a coin at rest, at a single instant, the following counterfactual would be true: were the coin not heads up, then it would not be tails down. Again, though, this not a causal connection, and again the described principle predicts just this. Some overarching property, F (being twosided), of a system, $S$ (the coin), that in concert with some part of the system, s1 (the headsface), and its property, G (facing up), serves to necessitate that the property, $\mathrm{H}$ (facing down), is instantiated, by some part of the system, s2 the tails face), that is not identical to s1. Thus, the instantiation of $\mathrm{F}$ and $\mathrm{G}$ (individually or jointly) are not among the causes of s2 instantiating $\mathrm{H}$.

And, is as clear, this principle will pose a problem for Baron and Miller for it is of the exact form of their case. There is some property (being in an entangled state) of a system, $S$ (the pair of particles), that in concert with some part of the system (particle 1) and its property G (spin up(/down)), serves to necessitate that the property $\mathrm{H}$ (spin down(/spin up)) is instantiated by some part of the system (particle 2) that is not identical to particle 1. As such, the instantiation of being in an entangled state and being spin up (/spin down) are not among the causes of P2 instantiating spin down (/spin up).

So, here is where I think we find ourselves. RED-BLUE* presents a useful comparison with the case Baron and Miller generate. RED-BLUE* isn't causal. It is of the same form as Baron and Miller's case. That being so, Baron and Miller's case isn't causal either. In this subsection, I've tried to offer a general principle that can be used to modify the Lewisian counterfactual model of causation to capture that finding, and to argue that the modification is itself intuitive. In the current paper at least, that's the end of the road for the positive story that Baron and Miller tell. But, as I noted at the outset, I think that there are independent

\footnotetext{
${ }^{4}$ The choice of directions 'up' and 'down' is irrelevant here. All that we require is that if the coin lands with heads facing D, then tails faces not-D. 'Direction D' and 'direction not D' are rather unlovely terms, though, so I persist with the more homely 'up' and 'down' in the case itself.
} 
reasons to think that we can have causation in a timeless world. Let me now turn to that positive story.

\section{Baron and Miller on Tallant}

According to my earlier temporal part (Tallant, 2008), we can show that causation without time is possible by considering certain possibilities that are generated via considerations of recombination and demonstrating that what we have are causal counterfactuals. For this section, I will once again follow Baron and Miller in endorsing a counterfactual theory of causation, with the emendations noted in sections 2 ('Distinctness). In this section I'll proceed as follows. I'll start by giving Baron and Miller's presentation of my earlier argument. I'll grant them all of their assumptions and show, in section 3.1, that there is an argument very close to that which I presented (in my 2008) that shows that we can have causation without time.

To begin, let us follow Baron and Miller (and my earlier temporal part) and start with a standard B-theoretic world, $\mathrm{wB}$, where the reality of time consists of the existence of the Brelations, earlier than, later than, and simultaneous with. This gives us our starting world. From this world, via standard principles of recombination, simply "remove" the B-relations from $w B$, leaving the rest of the world intact. Call the resulting world wS. Note, then, that $\mathrm{wS}$, will support the same counterfactuals as wB. After all, wS has the same structure. The only difference between $\mathrm{wS}$ and $\mathrm{wB}$ is that the former and not the latter lacks temporal relations; relations that are sufficient for the reality of time.

As Baron and Miller (2015a: 30) put it:

In what respects is $w S$ just like $w B$ except that $w B$ contains B-relations while wS not? At a minimum, we assume (i) for every slice in $w B$, there is a slice in $w S$ an intrinsic duplicate of that slice, and (ii) there is no slice in wS that is not an intrinsic duplicate of some slice in $\mathrm{wB}$, and (iii) there is a 1:1 mapping of the slices. If (i), (ii), and (iii) hold, then wS is what we will call a minimal intrinsic slice duplicate: it contains an intrinsic duplicate of every slice in $\mathrm{wB}$, and contains no more slices than that. There are two further assumptions one might make to secure the closeness of $\mathrm{wS}$ one might assume that the 1:1 mapping function $\mathrm{F}$ between slices of $\mathrm{wB}$ and $\mathrm{wS}$ orderpreserving. That is, the function maps each slice into its counterpart in a way that 
preserves the ordering of slices between $\mathrm{wB}$ and $\mathrm{wS}$. Second, one could assume that wB and wS have the same laws.

Granted all of that, wS will support the same counterfactuals as wB (cf. Tallant 2008: 120-1, Baron and Miller (2015a: 30). If wS supports the same counterfactuals as $w B$, and the counterfactual theory of causation is correct, then we have causation at wS (for we have causation at wB). But since wS has, by stipulation, no B-relations, then time is not real at wS. Hence wS is a world that lacks time but includes causation.

Here is Baron and Miller's (2015a: 30) formal reconstruction of my argument:

(1) B-relations are necessary and sufficient for the existence of temporal relations.

(2) wB contains B-relations

(3) wB contains temporal relations (from 1 and 2)

(4) wB contains causal relations (stipulation)

(5) wS is just like wB except that wS does not contain B-relations

(6) $\mathrm{wS}$ contains no temporal relations (from 5)

(7) wS supports the same counterfactuals as wB

(from 5)

In response, Baron and Miller (2015a: 30-1) look to reject (7). Their concern focuses on whether or not the laws at wB are the same as the laws at wB. They express this concern in the form of a dilemma.

Do wB and wS share the same laws of nature? If the answer is "yes", then B-relations (/time) makes no physical difference to the universe. For if wB and wS have the exact same laws and distribution of matter, then the B-relations simply make no difference. Say Baron and Miller (2015a: 30-1—emphasis mine):

That is hard to swallow. It is not that the presence/absence of time must make a physical difference to the universe by appearing in the laws of nature. Nor are we suggesting that any difference in the distribution of matters of fact in a world necessitates a difference in the laws in that world. Rather, recall that $\mathrm{wB}_{\mathrm{B}}$ and $\mathrm{wS}$ are intrinsic slice duplicates. The intrinsic matters of fact in $\mathrm{wS}$ are the same as in $\mathrm{wB}$. So the presence/absence of time can be making 
no difference to the intrinsic character of the slices, and no difference to the laws. So the question is, where else could the difference show up? Not in any experimental finding, since the slices are intrinsically the same. But if there is no empirical difference between the two worlds, then it is difficult to see what grounds we have for believing that time is physically relevant.

Suppose, instead, that we allow that the answer is "no": the laws of nature at wB and wS are not the same. It follows that (7) is false. Given a standard (Lewisian) model of counterfactuals, the laws of nature underpin counterfactual dependence. If the laws of nature at $\mathrm{wB}$ and $\mathrm{wS}$ differ, then it would follow that the counterfactuals supported by wB and wS differ.

\subsection{Response to Baron and Miller}

I suggest, here, that Baron and Miller leave open a very straightforward response. They are quite right that I (2008: 119) claim to be able to reconstruct the counterfactual dependencies of the actual world (assuming the actual world to be B-theoretic) at a timeless world. In that sense, my argument fails and they are right to reject it.

But, as promised at the outset, there is a nearby argument that promises to deliver the same verdict. I don't need as any part of an argument for the possibility of causation without time the premise that Baron and Miller have as their premise (5). Indeed, all that I require, to demonstrate the possibility of causation without time, is the following:

C1. $w B$ is a world at which causation is real

$\mathrm{C} 2$. $\mathrm{wS}$ is a world that can be 'generated' via recombination from $\mathrm{wB}$ and is timeless

C3. wS is a world at which counterfactual dependence obtains.

C4. From C2, C3 and the counterfactual theory of causation, wS is a world at which there is causation and no time

Now, again, I think that Baron and Miller are plausibly correct to put pressure on my claim that wB and wS support the same counterfactuals. But as C1-C4 collectively show, that doesn't matter. Tallant doesn't need that in order to establish the possibility of causation without time (though he may well need it for other reasons associated with the dialectic). 
To generate a case that demonstrates the possibility of causation without time, we need to borrow three elements from my (2008) discussion.

'Instants':

Consider, what we might think of as, a single instant. That is, a particular way that the world might be at any given time, a particular 3D arrangement of physical objects. The cricket ball is poised, millimetres away from the stumps having beaten the bat, the bathtub is full and just one more drop from the tap will send water cascading over the side, the water itself seems to form a column from the tap to the bath-but nothing moves. This single instant looks, if we could look at it, much as a paused video might. This is our single instant. Consider this instant to be world w: is time real in w? Seemingly not. There are no B-relations. (2008: 118)

'Ordering':

we order the instants according to the level of entropy at the macro-physical state across the entire instant. So our series has a high entropy state at one end, and a low entropy state at another. Since entropy (at the macroscopic level) increases towards what we think of as the future, this seems like an excellent way to order our series. (2008: 119)

'Timeless counterfactuals':

Consider the timeless worlds $\mathrm{w}, \mathrm{w} *$ and $\mathrm{w} * *$. These worlds are, explicitly, time-less: that is, there are no temporal relations over and above the causal relations. They are internally ordered according to entropic ordering relation stipulated [in 'Ordering']. Is there any reason to think that counterfactual dependence might not hold between various timeless instants? Not obviously. In w my ping occurs at t. My $\psi$ ing occurs at $t *$ In the relevantly close world $w *$, where I do not $\varphi$ at $t$, my $\psi$ ing does not occur at $t *$. In the relevantly close possible world $\mathrm{w} * *$ where I do $\varphi$ at $\mathrm{t}$, my $\psi$ ing occurs at $\mathrm{t} *$. In such a case there is clear counterfactual dependence. But do we have any reason to suppose that time is real? Not obviously. All that has been stipulated is that in certain worlds we have what looks to be counterfactual dependence across various instants. At all [relevant close] timeless worlds where I $\varphi$ at $t$, my $\psi$ ing occurs at $t *$. (2008: 121) 
If we put together 'instants', 'ordering' and 'Timeless counterfactuals', as well as the counterfactual theory of causation, then it follows that causation is possible in the absence of time. The counterfactuals described in Timeless Counterfactuals seem to be true. They are, for all that's been said, seemingly causal. Given 'instants' and 'ordering', the world described appears timeless. Thus, we seem to have causation in a timeless world.

No part of this statement of an argument for the possibility of causation without time requires that those counterfactuals be exactly the same as they would be at a world where time is real. That being so, there is a perfectly sensible reconstruction of my argument that is immune to Baron and Miller's criticism. My earlier mistake plausibly lies in thinking that we can get the exact same counterfactuals at $\mathrm{wS}$ as we get at $\mathrm{wB}$. But no such thing is required. So long as we can still preserve counterfactual dependence at wS (and the quoted elements of the earlier machinery should seem to get us that), then (assuming the counterfactual theory is true) we will have causation at wS.

Of course, there are a range of possible replies to this argument. One might, for instance, object to some aspect of the recombinatorial story. One might object that the counterfactual theory of causation is false. One might object that the B-theory is necessarily false, anyway, and so that the whole project starts off on bad foundations. All of these options could be explored.

However, I will not be exploring them here. As noted earlier in 3, my aim in this last section has been to demonstrate that Baron and Miller do not succeed in blocking an argument that is a nearby cousin of that I articulated and that can be recovered from the materials in his earlier paper. Since Baron and Miller are prepared to grant me each of 'instants', 'ordering', 'timeless counterfactuals' and the counterfactual theory of causation, I have done so too. Provided we are prepared to allow those concessions, it looks as if we have an argument to the conclusion that we may have causation in a timeless world.

\section{Conclusion}

Granted certain assumptions, causation with time seems coherent, though not for the reasons spelled out by Baron and Miller. As I explained at the outset, this is a useful result when it comes to the matter of reconstructing our manifest image of the world within a model of 
reality that is itself timeless. Indeed, it seems that if we are to defend a timeless model of reality, then we must engage in the project of reconstructing at least some of that manifest image, including some role for causation and causal judgements. How far we can extend that reconstruction is a question that must be explored elsewhere. Nonetheless, since preserving causation would seem to be an integral part of any such effort, the current project is an important one. ${ }^{5}$

\section{References:}

Barbour, J. (1994a). The timelessness of quantum gravity: I. The evidence from the classical theory. Classical Quantum Gravity, 11(12), 2853-2873.

Barbour, J. (1994b). The timelessness of quantum gravity: II. The appearance of dynamics in static configurations. Classical Quantum Gravity, 11(12), 2875-2897.

Barbour, J. (1999). The end of time. Oxford: Oxford University Press.

Barbour, J., \& Isham, C. (1999). On the emergence of time in quantum gravity. In J. Butterfield (Ed.), The arguments of time (pp. 111-168). Oxford: Oxford University Press.

Barker, S. 2003. 'Counterfactual Analyses of Causation: The Problem of Effects and Epiphenomena Revisited', Noûs, 37, 133-50

Baron, S. \& Miller, K. (2014). Causation in a Timeless World, Synthese, 181, 2867-2886

Baron, S. and Miller, K. (2015a). Causation sans Time, American Philosophical Quarterly, $52,27-40$

Baron, S. \& Miller, K. (2015b). What is Temporal Error Theory?, Philosophical Studies, 172, 2427-2444

\footnotetext{
${ }^{5}$ Some of the work on this paper was carried out during a visiting fellowship at the University of Sydney. I'm very grateful to everyone involved. I owe a particular debt to Dean Rickles, Kristie Miller, David BraddonMitchell and members of the graduate work in progress group. Thanks folks! I'm also very grateful to two referees for this journal for extremely helpful comments on an earlier version of this paper and to David Ingram for conversations about a very early draft of the ideas here.
} 
Carroll, J. (2002). 'Instantaneous motion', Philosophical Studies, 110, 49-67

Deutsch, D. (1997). The fabric of reality: The science of parallel universes and its implications. London: Penguin.

Healey, R. (2002). Can physics coherently deny the reality of time? In C. Callender (Ed.), Time, reality \& experience (pp. 293-316). Cambridge: Cambridge University Press.

Lewis, D. (1973). 'Causation', Journal of Philosophy, 70, 556-567

Lewis, D. (1986a). 'Counterfactual Dependence and Time's Arrow', in in Philosophical Papers, Vol. 2 New York: Oxford University Press, 32-52

Lewis, D. (1986b). On the Plurality of Worlds Oxford: Blackwell

Rovelli, C. (2004). Quantum gravity. Cambridge: Cambridge University Press.

Rovelli, C. (2007). The disappearance of space and time. In Dennis Dieks (Ed.), The ontology of spacetime (pp. 25-36). Amsterdam: Elsevier.

Tallant, J. (2008). What is it to ' $B$ "' a relation? Synthese, 162, 117-132 